\title{
Use of teflubenzuron alone and combined with Metarhizium anisopliae and Phenylacetonitrile as control agent against the desert locust, schistocerca gregaria (Forskal) (Orthoptera: acrididae)
}

\author{
Mahgoub M. Mohamed ${ }^{1}$, Hamadttu A. Elshafie ${ }^{2}$, and Magzoub O. Bashir ${ }^{3}$ \\ ${ }^{1}$ Plant Protection Directorate, P.O. Box 14, Khartoum north, Sudan \\ ${ }^{2}$ University of Khartoum, Faculty of Agric. Crops Protection Department, Shambat, Sudan \\ ${ }^{3}$ International Center of Insect Physiology and Ecology (ICIPE) Port Sudan, Sudan \\ Corresponding author:Hamadttu, A. Elshafie, e-mail: elshafie62@yahoo.com
}

\begin{abstract}
The efficacy of the Insect growth regulator (IGR) teflubenzuron (Nomolt 50 ULV) was evaluated against desert locust (DL) nymphs (3rd and 4th instars) during two seasons (2008 and 2009). All experiments were carried out under semi-field conditions, on irrigated plots planted with millet, and large cages $(2 \times 2 \times 1 \mathrm{~m})$ and bomas $(2 \times 6 \times 1 \mathrm{~m})$ were erected to enclose the test insects. The IGR was applied by means of a battery driven hand held (Micro ULVA) sprayer either as direct spray on the nymphs or as a simulated barrier treatment. The impact of the IGR on the feeding rate of the nymphs was investigated as well. The IGR was tested at the recommended dose (450 $\mathrm{ml} / \mathrm{ha})$, and at fractional doses $(225,112.5$ and $56.25 \mathrm{ml} / \mathrm{ha}$ ) in combination with Metarhizium anisopliae (Green Muscle ${ }^{\circledR}$ ) at $50 \mathrm{~g} / \mathrm{ha}$ and the pheromone Phenyl Aceto Nitrile (PAN) $98 \%$ at $10 \mathrm{ml} / \mathrm{ha}$. Diesel was used as a dilutant in all formulations. Nomolt $450 \mathrm{~m} / \mathrm{ha}$ resulted in $100 \%$ nymphal mortality in 20 days after treatment. The fractional doses of the IGR combined with the pheromone PAN, proved to be effective in the manipulation of nymphs, $100 \%$ mortality was obtained in 21, 20 and 21 days after treatment, in both seasons of the study. The fractional doses of the IGR combined with Metarhizium GM resulted in 100\% mortality within 14 days of the treatment. Tests in bomas using barrier treatment in the $1^{\text {st }}$ season of the study revealed that the recommended dose of Nomolt applied on the nymphs resulted in $72.5,88.5,89$ and $53 \%$ mortality after the $1^{\text {st }}, 6^{\text {th }}, 12^{\text {th }}$ and $24^{\text {th }}$ day after treatment respectively. Percentage mortality of $78.5,68.5$, 74 and 61.5 were achieved in the $2^{\text {nd }}$ season. The Nomolt either alone or in combined with PAN and GM revealed a clear negative impact on the feeding rate of nymphs.
\end{abstract}

Key words: Metarhizium anisopliae, Teflubenzuron, Phenylacetonitrile, Pheromone, Desert Locust, entomopathogenic fungus

\section{INTRODUCTION}

The desert locust Schistocerca gregaria (Forskal) is one of the most important pests due to its extensive and catastrophic damage to crops in large parts of Africa and Asia. During the upsurge (years 20032005) which occurred in West Africa, nearly 130,000 $\mathrm{km}^{2}$ were treated by ground and aerial operations. The costs of fighting this upsurge was estimated by the FAO to have exceeded US $\$ 400$ million and harvest losses were valued at up to US\$2.5 billion which had disastrous effects on the food security situation in West Africa (Ceccato, et al., 2007). The major control strategy is based on the use of insecticides. All the insecticides sprayed had some potentially negative environmental effects. Rembold (1994) adverted to the rapidly increasing insect tolerance against any type of neurotoxic insecticide, and all insecticides given their wide spectrum of action undoubtedly had substantial side-effects on the non-target fauna (Müller, 1988). Thus, alternative to these harmful pesticides must be found. Peveling, et al. (1994) showed that, there was no evidence of serious side-effects of the alternative control agents tested so far on epigeal arthropods when compared with conventional insecticides. One of these promising alternatives are insect growth regulators (IGRs) also called third-generation insecticides. The IGRs are pesticides that affect the development, 
reproduction, or metamorphosis of the target insect, thus stopping the life cycle and infestation. Chitin synthesis inhibitors (CSIs) have proven to be suitable replacements in locust control for organochlorine insecticides under field conditions. Teflubenzuron was found to be effective in barrier trials against Locusta migratoria capito, and has also been shown to be very effective in controlling hopper bands of Schistocerca gregaria (Lecoq, et al., 1988). Also one of the alternatives which have attracted increasing interest was the entomopathogenic fungus Metarhizium anisopliae var. acridum, because of its high specificity and very little adverse effect on the environment. Metarhizium anisopliae, is now produced commercially with trade names such as Green Muscle and Green Guard. The use of Green Muscle represents a new approach to locusts and grasshoppers control. The scientists at International Center of Insect Physiology and Ecology (ICIPE) have identified and synthesized a specific locust pheromone, or chemical signal, that can be used against young locusts. The pheromone Phenylacetonitrile (PAN) induced solitary behavior, confused and disoriented insects, some lost their appetite altogether, while others turned cannibal and ate one another any survivors were easy prey for predators.

The present work is carried out with the main following objectives:

- To elucidate the efficacy of the IGR Nomolt (in recommended and fractional doses) on the nymphs of the desert locust.

- To study the compatibility of the Nomolt with Metarhizium and the pheromone PAN.

- To test the efficacy of the Nomolt when applied in a simulated barrier treatment.

\section{MATERIALS AND METHODS}

Site: The trials were carried out in an area at Salloum farm (19 $3855 \mathrm{~N}$ x $371537 \mathrm{E}) 28 \mathrm{~km}$ south west of Port Sudan, during two consecutive seasons (December 2007- June 2008 and December 2008June 2009).

Experimental insects: Adults of desert locust were obtained from Tokar Delta and mass reared at the International Center of Insect Physiology and Ecology (ICIPE) field station, Port Sudan. Cages (1x1x0.35 m) fitted with wire mesh and a movable window from cloth, to facilitate cleaning and feeding, were used for the purpose. The cages stand on poles one meter from the ground, 24 cups $(10 \times 12 \mathrm{~cm})$ filled with moist soil are fitted in each cage for egg-laying. Locusts were fed on food plants such as Pennisetum typhoidium and Heliotropium spp. The hatching nymphs were removed from the cups $(10 \times 12 \mathrm{~cm})$ to cages $(1 \times 1 \times 0.7 \mathrm{~m})$ fitted with cloth and wire mesh and supplied with food. The $3^{\text {rd }}$ and $4^{\text {th }}$ instars of desert locust nymphs were used in the trials. Some of these nymphs were transferred into large cages $(4 \times 2 \times 2 m)$ made from steel and wire mesh where they were kept for the continuity of desert locust rearing.

Control agents: The IGR teflubenzuron (Nomolt 50 ULV) was tested at the recommended dose $(450 \mathrm{ml} / \mathrm{ha})$, and in fractional doses $(225,112.5$ and $56.25 \mathrm{ml} / \mathrm{ha}$ ) alone and combined with Metarhizium anisopliae (Green Muscle ${ }^{\circledR}$ ) 50g/ha (recommended dose) and the pheromone Phenylacetonitrile (PAN) $98 \% 10 \mathrm{ml} / \mathrm{ha}$ (recommended dose). Diesel was used as a dilutant in all formulations.

Experimental set-up : Trials were carried out under semi - field conditions on irrigated plots $(2 \times 2 \mathrm{~m})$ planted with millet and large bomas and cages were erected. Ten treatments replicated three times as listed below, were applied to randomly selected plots.

i. Nomolt $450 \mathrm{ml} / \mathrm{ha}$ (recommended dose).

ii. Nomolt $225 \mathrm{ml}+$ diesel $225 \mathrm{ml}+\mathrm{GM} 11.5 \mathrm{~g}$.

iii. Nomolt $112.5 \mathrm{ml}+$ diesel $337.5 \mathrm{ml}+\mathrm{GM} 11.5 \mathrm{~g}$.

iv. Nomolt $56.25 \mathrm{ml}+$ diesel $393.75 \mathrm{ml}+\mathrm{GM} 11.5 \mathrm{~g}$.

v. Nomolt $225 \mathrm{ml}+$ diesel $225 \mathrm{ml}+$ PAN4.5ml .

vi. Nomolt $112.5 \mathrm{ml}+$ diesel $337.5 \mathrm{ml}+$ PAN4.5ml.

vii. Nomolt $56.25 \mathrm{ml}+$ diesel $393.75 \mathrm{ml}+$ PAN4.5ml.

viii. Untreated Control.

The spraying technique: Hand held sprayer Micro ULVA, spinning disc type, operated by battery with tank capacity of one liter, was used for spraying the formulations. The calibration, spraying techniques and the parameters used for the pesticides spraying were as follows: The flow rate $=30 \mathrm{ml} /$ minute, height of sprayer nozzle $=0.5$ meter, disc speed $=7500$ $8000 \mathrm{rpm}$, track space $=10 \mathrm{~m}$, the speed $=4 \mathrm{~km} / \mathrm{hour}$, Volume application rate $(V A R)=450 \mathrm{ml} / \mathrm{ha}$.

\section{Methods of application}

Direct spraying: Large cages $(2 \times 2 \times 1 \mathrm{~m})$ made from wood and wire mesh, were erected on irrigated plots planted with millet. The $3^{\text {rd }}$ and $4^{\text {th }}$ instars were released before $24 \mathrm{hrs}$ of the treatments. In each 
cage 75 nymphs were placed and then treated with different doses of the IGR Nomolt alone and combined with different formulations to evaluate their efficacy in controlling these nymphs.

Barrier spraying: Large bomas $(2 \times 6 \times 1 \mathrm{~m})$ were erected on irrigated plots planted with millet and the IGR Nomolt 450ml/ha (recommended dose) was applied in a localized strip within the boma, i.e. the treated strip was $2 \times 2$ meter, while the untreated area was $4 \times 2$ meter. The reared stages of desert locust (3rd and 4th instars) starved for 24 hours were released in the treated bomas and left for $24 \mathrm{hrs}$. The nymphs were then removed to untreated cages for observations. This process was repeated 6, 12 and 24 days after the initial treatment.

Mortality inspection and control efficiency : Inspection was done daily for all cages. Dead insects due to treatment effected by the Nomolt or to cannibalism were picked out of the cage and recorded. Mortality was calculated for 21 days after treatment. Cadavers were collected for mycosis observation, from cages in which insects were treated with GM. Ten cadavers were incubated in Petri dishes containing wet filter paper and left at room temperature to trace the fungal sporulation.

The control efficiency of the different treatments was calculated using the formula of Henderson and Tilton (1955):

Control efficiency $=\left\{1-\left(\frac{T a}{\tau b} \times \frac{C_{b}}{C_{a}}\right) \times 100\right\}$

Where $\mathrm{Tb}$ and $\mathrm{Cb}=$ density before treatment in treated and control plots respectively; this is fixed; Ta and $\mathrm{Ca}=$ density after treatment in treated and control plots respectively. Control efficiency means were used to draw graphics.

Meteorological data: Relative humidity, temperature and wind speed were daily observed and reported every 2 hours. Mean ( \pm STD) temperatures $(T)$, relative humidity $(\mathrm{RH})$ and wind speed (WS) meter/second during the experiments were as follows:

Season I: $\mathrm{T} \min =25.19 \pm 2.48^{\circ} \mathrm{C}, \mathrm{T} \max =39.81 \pm$ $4.34^{\circ} \mathrm{C}, \mathrm{RH} \min =19.71 \pm 10.81 \%, \mathrm{RH} \max =57.90 \pm$
$18.83 \%$, WS $\min =1.76 \pm 0.66 \mathrm{~m} / \mathrm{s}, \mathrm{WS} \max =3.39 \pm$ $0.83 \mathrm{~m} / \mathrm{s}$.

Season II: $\mathrm{T} \min =18.67 \pm 3.91^{\circ} \mathrm{C}, \mathrm{T} \max =35.81 \pm$ $3.31^{\circ} \mathrm{C}, \mathrm{RH} \min =11.86 \pm 17.25 \%, \mathrm{RH} \max =48.42 \pm$ $27.09 \%$, WS $\min =0.21 \pm 0.54 \mathrm{~m} / \mathrm{s}$, WS $\max =2.79 \pm$ $0.46 \mathrm{~m} / \mathrm{s}$.

Statistical analysis: The results were analyzed by analysis of variance (ANOVA) using SPSS software package vers.15. The Least Significance difference (LSD) was used to determine differences between treatments means.

\section{RESULTS}

Effect of the recommended dose of the IGR Nomolt and the fractional doses in combination with PAN on the nymphs: In season 1 (year 2008), the recommended dose of the Nomolt $450 \mathrm{ml} / \mathrm{ha}$ resulted in a $100 \%$ mortality of the test insects on the $20^{\text {th }}$ days after treatment and the fractional doses of the Nomolt: $225 \mathrm{ml} / \mathrm{ha}, 112.5 \mathrm{ml} / \mathrm{ha}$ and $56.25 \mathrm{ml} / \mathrm{ha}$ combined with PAN $10 \mathrm{ml} / \mathrm{ha}$ (rec. dose) resulted in $100 \%$ mortality on the 21,20 and 21 days after treatment respectively. Percentage mortalities of 74.2 $85.8,88.9$ and $84.4 \%$ were respectively due to treatments. Cannibalism among test insects accounted for $25.8 \% 14.2,11.1$ and $15.6 \%$ mortality respectively in the different treatment was achieved (Table 1).

The control efficiency of the control agents under investigation are shown in Figure 1.

In season 2 (year 2009) the recommended dose of the Nomolt resulted in a $100 \%$ mortality of the test insects on the $16^{\text {th }}$ days after treatment. Percentage mortality of 86.7 was achieved and $13.3 \%$ was attributed to cannibalism, while the fractional doses of the Nomolt combined with PAN proved their efficacy on desert locust manipulation. Hundred percentage mortality was achieved in 17, 15 and 14 days after treatment respectively. Of this 78.7, 83.1and $76 \%$ was due to treatment effected by the Nomolt and $21.3,16.9$ and $24 \%$ due to cannibalism (Table 2). The control efficiency of the control agents under investigation are shown in Figure 2. 
Agric. Biol. J. N. Am., 2011, 2(9): 1293-1303

Table 1. \% Mortality of various doses of the IGR Nomolt combined with pheromone PAN on the nymphal instars of the DL (year/2008)

\begin{tabular}{|c|c|c|c|c|c|c|c|c|c|c|c|}
\hline \multirow{2}{*}{ Treatment } & \multicolumn{11}{|c|}{$\%$ Mortality at days after treatment } \\
\hline & 1 & 3 & 5 & 7 & 9 & 11 & 13 & 15 & 17 & 19 & 21 \\
\hline \multirow[t]{2}{*}{$\begin{array}{l}\text { Nomolt } \\
450 \mathrm{ml} / \mathrm{ha}\end{array}$} & $\begin{array}{c}.11 \pm \\
0.58\end{array}$ & $\begin{array}{c}7.11 \pm \\
3.06\end{array}$ & $\begin{array}{c}20.44 \pm \\
0.58\end{array}$ & $\begin{array}{c}22.67 \pm \\
2.89\end{array}$ & $\begin{array}{c}37.33 \pm \\
1.53\end{array}$ & $\begin{array}{c}54.22 \pm \\
1.00\end{array}$ & $\begin{array}{c}68.89 \\
\pm \\
3.46\end{array}$ & $\begin{array}{c}86.22 \\
\pm \\
1.53\end{array}$ & $\begin{array}{c}93.78 \\
\pm \\
0.58\end{array}$ & $\begin{array}{c}97.78 \\
\pm \\
0.58\end{array}$ & $\begin{array}{l}100 \pm \\
0.00\end{array}$ \\
\hline & $(2.22)$ & $(4.89)$ & $\begin{array}{c}(15.11 \\
)\end{array}$ & $(16.89)$ & $(22.67)$ & $(25.78)$ & & & & & \\
\hline \multirow[t]{2}{*}{$\begin{array}{l}\text { Nomolt } \\
225 \mathrm{ml} / \mathrm{ha}+ \\
\text { PAN }\end{array}$} & $\begin{array}{c}2.67 \pm \\
0.00\end{array}$ & $\begin{array}{c}14.67 \\
\pm \\
0.58\end{array}$ & $\begin{array}{c}30.67 \pm \\
0.58\end{array}$ & $\begin{array}{c}37.33 \pm \\
2.89\end{array}$ & $\begin{array}{c}57.78 \pm \\
1.73\end{array}$ & $\begin{array}{c}67.56 \pm \\
3.46\end{array}$ & $\begin{array}{c}80.44 \\
\pm \\
0.58\end{array}$ & $\begin{array}{c}90.67 \\
\pm \\
5.77 \\
\end{array}$ & $\begin{array}{c}97.78 \\
\pm \\
4.16 \\
\end{array}$ & $\begin{array}{c}99.11 \\
\pm \\
1.73 \\
\end{array}$ & $\begin{array}{l}100 \pm \\
1.15\end{array}$ \\
\hline & (1.33) & $(6.22)$ & $\begin{array}{c}(11.56 \\
)\end{array}$ & $(12.00)$ & $(13.78)$ & $(14.22)$ & & & & & \\
\hline \multirow[t]{2}{*}{$\begin{array}{l}\text { Nomolt } \\
112.5 \mathrm{ml} / \mathrm{ha}+ \\
\text { PAN }\end{array}$} & $\begin{array}{c}1.78 \pm \\
0.58\end{array}$ & $\begin{array}{c}14.67 \\
\pm \\
3.00 \\
\end{array}$ & $\begin{array}{c}25.33 \pm \\
3.79\end{array}$ & $\begin{array}{c}33.78 \pm \\
2.31\end{array}$ & $\begin{array}{c}47.56 \pm \\
1.73\end{array}$ & $\begin{array}{c}59.56 \pm \\
3.21\end{array}$ & $\begin{array}{c}76.44 \\
\pm \\
2.08 \\
\end{array}$ & $\begin{array}{c}89.78 \\
\pm \\
1.00 \\
\end{array}$ & $\begin{array}{c}97.78 \\
\pm \\
0.58 \\
\end{array}$ & $\begin{array}{c}99.11 \\
\pm \\
0.58 \\
\end{array}$ & $\begin{array}{l}100 \pm \\
0.00\end{array}$ \\
\hline & (1.33) & $(4.44)$ & $(7.11)$ & $(8.44)$ & $(11.11)$ & & & & & & \\
\hline \multirow[t]{2}{*}{$\begin{array}{l}\text { Nomolt } \\
56.25 \mathrm{ml} / \mathrm{ha}+ \\
\text { PAN }\end{array}$} & $\begin{array}{c}4.89 \pm \\
0.58\end{array}$ & $\begin{array}{c}11.56 \\
\pm \\
1.73 \\
\end{array}$ & $\begin{array}{c}17.33 \pm \\
1.15\end{array}$ & $\begin{array}{c}20.00 \pm \\
1.73\end{array}$ & $\begin{array}{c}27.11 \pm \\
1.00\end{array}$ & $\begin{array}{c}35.11 \pm \\
1.00\end{array}$ & $\begin{array}{c}58.22 \\
\pm \\
2.00 \\
\end{array}$ & $\begin{array}{c}74.67 \\
\pm \\
2.00 \\
\end{array}$ & $\begin{array}{c}85.33 \\
\pm \\
2.00 \\
\end{array}$ & $\begin{array}{c}96.44 \\
\pm \\
1.00\end{array}$ & $\begin{array}{l}100 \pm \\
1.15\end{array}$ \\
\hline & $(3.56)$ & $(7.56)$ & $\begin{array}{c}(10.22 \\
)\end{array}$ & $(11.56)$ & (15.56) & & & & & & \\
\hline Control & $\begin{array}{c}1.78 \pm \\
0.58\end{array}$ & $\begin{array}{c}3.11 \pm \\
0.58\end{array}$ & $\begin{array}{l}3.11 \pm \\
0.00\end{array}$ & $\begin{array}{c}3.56 \pm \\
0.00\end{array}$ & & & & & & & \\
\hline
\end{tabular}

\pm Standard deviation (SD)

( ) \% cannibalized nymphs observed after treatment

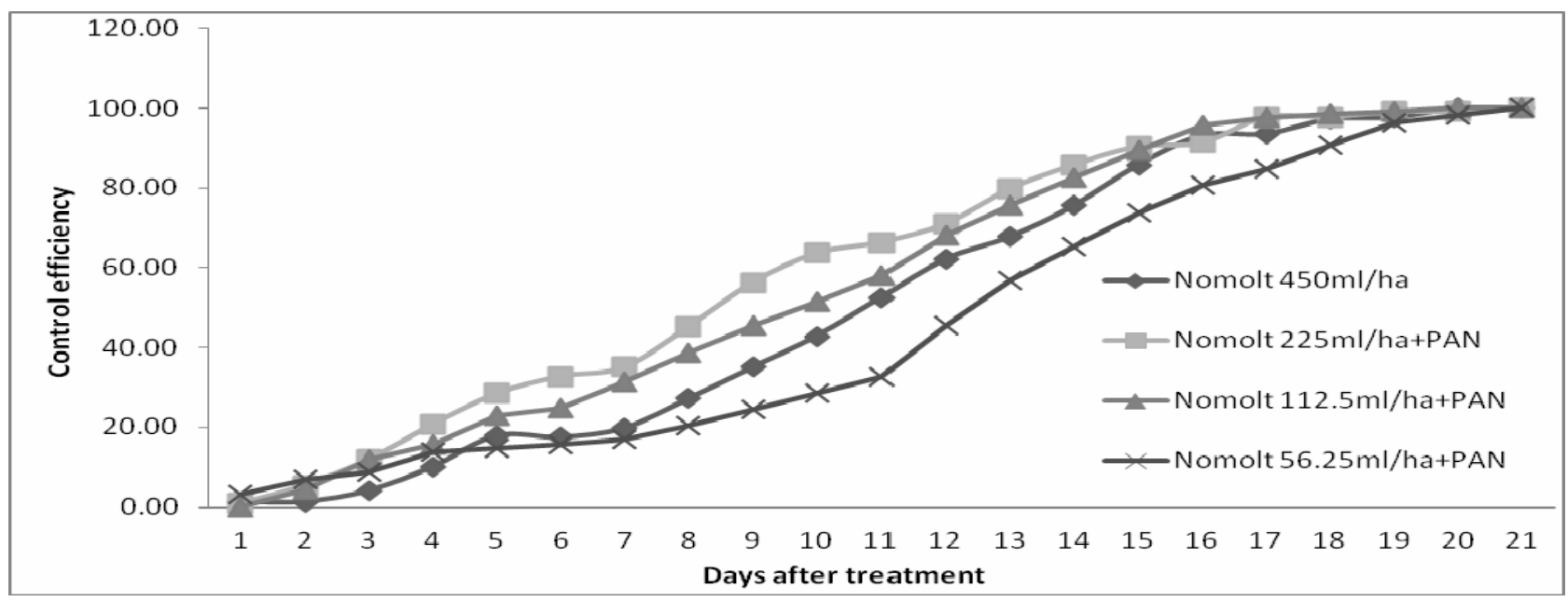

Fig 1. Mean control efficiency of desert locust nymphs treated with different doses of the IGR Nomolt plus PAN10ml/ha (year/2008) 
Agric. Biol. J. N. Am., 2011, 2(9): 1293-1303

Table 2. Percentage mortality of various doses of the IGR Nomolt combined with pheromone PAN on the nymphal instars of the desert locust (year/2009)

\begin{tabular}{|c|c|c|c|c|c|c|c|c|c|c|c|}
\hline \multirow{2}{*}{ Treatment } & \multicolumn{11}{|c|}{$\%$ Mortality at days after treatment } \\
\hline & 1 & 3 & 5 & 7 & 9 & 11 & 13 & 15 & 17 & 19 & 21 \\
\hline \multirow[t]{2}{*}{$\begin{array}{l}\text { Nomolt } \\
450 \mathrm{ml} / \mathrm{ha}\end{array}$} & $\begin{array}{c}7.56 \pm \\
3.06\end{array}$ & $\begin{array}{c}23.11 \pm \\
3.51\end{array}$ & $\begin{array}{c}46.67 \\
\pm \\
4.93\end{array}$ & $\begin{array}{c}65.33 \pm \\
2.65\end{array}$ & $\begin{array}{c}73.78 \pm \\
4.16\end{array}$ & $\begin{array}{c}81.78 \pm \\
1.53\end{array}$ & $\begin{array}{c}91.11 \\
\pm \\
2.31\end{array}$ & $\begin{array}{c}97.78 \\
\pm \\
1.53\end{array}$ & $\begin{array}{l}100 \pm \\
0.00\end{array}$ & & \\
\hline & $(3.11)$ & $(7.56)$ & $(9.33)$ & $(12.89)$ & $(12.89)$ & $(13.33)$ & & & & & \\
\hline \multirow[t]{2}{*}{$\begin{array}{l}\text { Nomolt } \\
225 \mathrm{ml} / \mathrm{ha}+\mathrm{PAN}\end{array}$} & & $\begin{array}{c}10.22 \pm \\
3.00\end{array}$ & $\begin{array}{c}23.11 \\
\pm \\
2.65\end{array}$ & $\begin{array}{c}38.67 \pm \\
4.51\end{array}$ & $\begin{array}{c}67.56 \pm \\
2.52\end{array}$ & $\begin{array}{c}84.00 \pm \\
3.21\end{array}$ & $\begin{array}{c}92.00 \\
\pm \\
3.61\end{array}$ & $\begin{array}{c}97.33 \\
\pm \\
1.73\end{array}$ & $\begin{array}{c}100 \pm \\
1.15\end{array}$ & & \\
\hline & & $(5.33)$ & $(7.56)$ & $(9.78)$ & $(14.22)$ & $(17.33)$ & $\begin{array}{c}(18.22 \\
)\end{array}$ & $\begin{array}{c}(20.00 \\
)\end{array}$ & $\begin{array}{c}(21.33 \\
)\end{array}$ & & \\
\hline \multirow[t]{2}{*}{$\begin{array}{l}\text { Nomolt } \\
112.5 \mathrm{ml} / \mathrm{ha}+ \\
\text { PAN }\end{array}$} & $\begin{array}{c}1.33 \pm \\
1.00\end{array}$ & $\begin{array}{c}17.33 \pm \\
3.61\end{array}$ & $\begin{array}{c}38.22 \\
\pm \\
0.58\end{array}$ & $\begin{array}{c}52.89 \pm \\
1.53\end{array}$ & $\begin{array}{c}83.11 \pm \\
2.52\end{array}$ & $\begin{array}{c}96.00 \pm \\
2.08\end{array}$ & $\begin{array}{c}98.22 \\
\pm \\
0.58\end{array}$ & $\begin{array}{l}100 \pm \\
1.73\end{array}$ & & & \\
\hline & $(0.44)$ & $(4.00)$ & $(9.78)$ & $(14.22)$ & $(16.00)$ & $(16.89)$ & & & & & \\
\hline \multirow[t]{2}{*}{$\begin{array}{l}\text { Nomolt } \\
56.25 \mathrm{ml} / \mathrm{ha}+ \\
\text { PAN }\end{array}$} & & $\begin{array}{c}16.44 \pm \\
4.51\end{array}$ & $\begin{array}{c}35.11 \\
\pm \\
4.51 \\
\end{array}$ & $\begin{array}{c}56.00 \pm \\
1.53\end{array}$ & $\begin{array}{c}79.11 \pm \\
1.53\end{array}$ & $\begin{array}{c}90.22 \pm \\
5.20\end{array}$ & $\begin{array}{c}97.78 \\
\pm \\
2.31 \\
\end{array}$ & $\begin{array}{l}100 \pm \\
0.00\end{array}$ & & & \\
\hline & & $(7.11)$ & $\begin{array}{c}(14.22 \\
)\end{array}$ & $(20.44)$ & $(24.00)$ & & & & & & \\
\hline Control & $\begin{array}{c}1.33 \pm \\
1.00\end{array}$ & $\begin{array}{c}3.11 \pm \\
0.58\end{array}$ & $\begin{array}{c}3.56 \pm \\
0.00\end{array}$ & & & & & & & & \\
\hline
\end{tabular}

\pm Standard deviation (SD)

( ) \% cannibalised nymphs observed after treatment

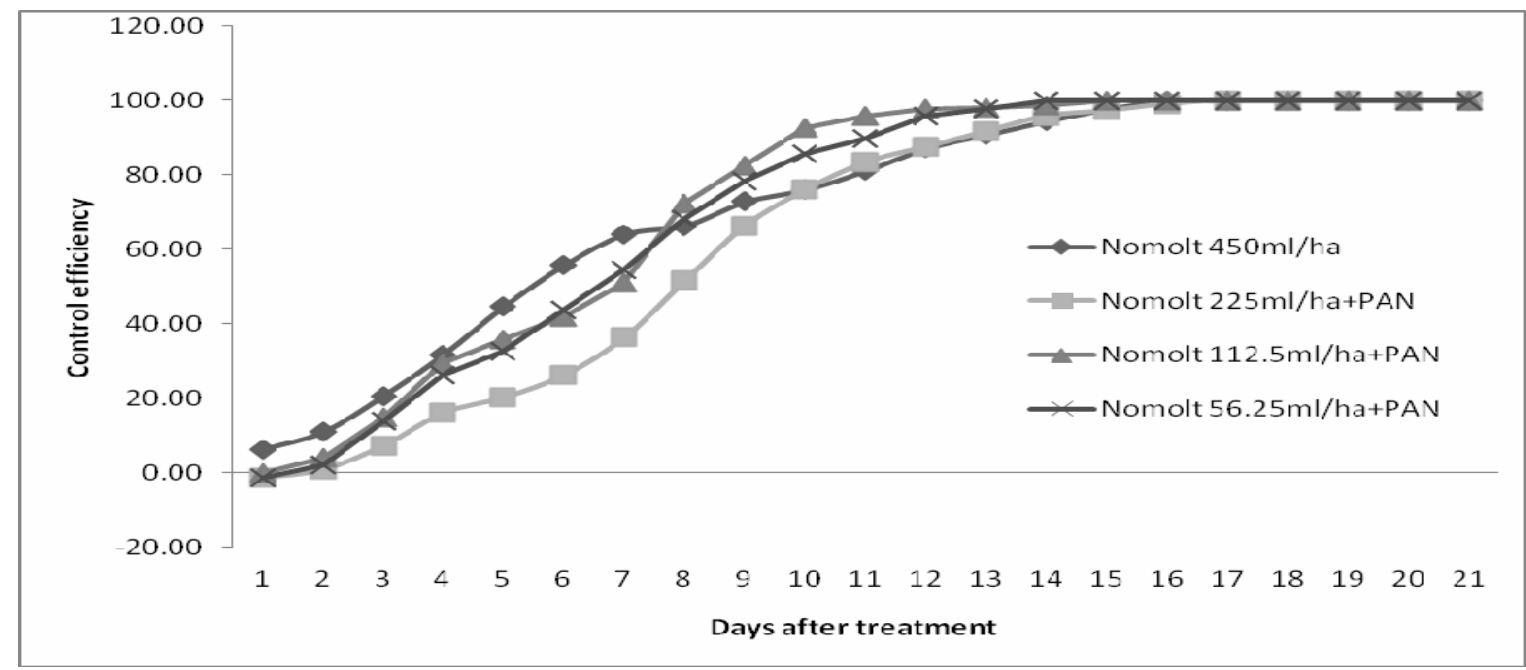

Fig 2. Mean control efficiency of desert locust nymphs treated with different doses of the IGR Nomolt plus PAN10ml/ha (year/2009)

All the hoppers in the control moulted successfully to the next instars within seven days post-treatment, however, none of those sprayed with IGR Nomolt did.

The Nomolt symptoms are characterized by hoppers dying while attempting to moult and they were deformed with missing limbs, soft to the touch and incapable of normal movement (Plate 2). Cannibalism and deformation in DL nymphs were very clear in treated cages with the Nomolt combined with PAN (Plate 3). 
The fractional doses of the Nomolt combined with pheromone PAN were effective and there was no significant difference compared to the recommended dose of the Nomolt.

Effect of the fractional doses of the Nomolt combined with Metarhizium: In season 1 the fractional doses of Nomolt (225, 112.5 and
$56.25 \mathrm{ml} / \mathrm{ha}$ ) combined with GM, 100\% mortality was achieved in 14 days, with no significant difference when different formulations were used. Out of this $100 \%$ mortality, $79.1,88$ and $88.4 \%$ was due to treatment effected by the Nomolt plus GM and 20.9, 12 and $11.6 \%$ due to cannibalism respectively (Table 3). The control efficiency of the control agents are illustrated in Figure 3.

Table 3. \% Mortality of various doses of the IGR Nomolt combined with GM on the nymphal instars of the desert locust (year/2008)

\begin{tabular}{|c|c|c|c|c|c|c|c|c|c|c|c|}
\hline \multirow{2}{*}{ Treatment } & \multicolumn{11}{|c|}{$\%$ Mortality at days after treatment } \\
\hline & 1 & 3 & 5 & 7 & 9 & 11 & 13 & 15 & 17 & 19 & 21 \\
\hline \multirow[t]{2}{*}{$\begin{array}{l}\text { Nomolt } \\
450 \mathrm{ml} / \mathrm{ha}\end{array}$} & $\begin{array}{c}3.11 \pm \\
0.58\end{array}$ & $\begin{array}{l}7.11 \pm \\
3.06\end{array}$ & $\begin{array}{c}20.44 \pm \\
0.58\end{array}$ & $\begin{array}{c}22.67 \pm \\
2.89 \\
\end{array}$ & $\begin{array}{c}37.33 \pm \\
1.53 \\
\end{array}$ & $\begin{array}{c}54.22 \pm \\
1.00 \\
\end{array}$ & $\begin{array}{c}68.89 \pm \\
3.46 \\
\end{array}$ & $\begin{array}{c}86.22 \pm \\
1.53 \\
\end{array}$ & $\begin{array}{c}93.78 \pm \\
0.58\end{array}$ & $\begin{array}{c}97.78 \pm \\
0.58 \\
\end{array}$ & $\begin{array}{l}100 \pm \\
0.00 \\
\end{array}$ \\
\hline & $(2.22)$ & $(4.89)$ & $(15.11)$ & $(16.89)$ & $(22.67)$ & $(25.78)$ & & & & & \\
\hline \multirow{2}{*}{$\begin{array}{l}\text { Nomolt } \\
225 \mathrm{ml} / \mathrm{ha}+ \\
\text { GM }\end{array}$} & $\begin{array}{c}12.89 \pm \\
2.31\end{array}$ & $\begin{array}{c}19.11 \pm \\
0.58\end{array}$ & $\begin{array}{c}35.11 \pm \\
1.53\end{array}$ & $\begin{array}{c}52.00 \pm \\
1.53\end{array}$ & $\begin{array}{c}69.33 \pm \\
7.21\end{array}$ & $\begin{array}{c}81.33 \pm \\
3.51\end{array}$ & $\begin{array}{c}97.33 \pm \\
2.89\end{array}$ & $\begin{array}{l}100 \pm \\
0.00\end{array}$ & & & \\
\hline & $(8.00)$ & $(13.33)$ & $(19.56)$ & $(19.56)$ & $(20.89)$ & & & & & & \\
\hline \multirow{2}{*}{$\begin{array}{l}\text { Nomolt } \\
112.5 \mathrm{ml} / \mathrm{ha}+ \\
\text { GM }\end{array}$} & $\begin{array}{c}11.56 \pm \\
3.21\end{array}$ & $\begin{array}{c}20.44 \pm \\
1.53\end{array}$ & $\begin{array}{c}29.78 \pm \\
1.00\end{array}$ & $\begin{array}{c}42.67 \pm \\
1.53\end{array}$ & $\begin{array}{c}70.22 \pm \\
1.53\end{array}$ & $\begin{array}{c}82.67 \pm \\
1.00\end{array}$ & $\begin{array}{c}96.44 \pm \\
2.65 \\
\end{array}$ & $\begin{array}{c}100 \pm \\
0.00\end{array}$ & & & \\
\hline & $(4.00)$ & $(7.11)$ & $(9.33)$ & $(12.00)$ & & & & & & & \\
\hline \multirow{2}{*}{$\begin{array}{l}\text { Nomolt } \\
56.25 \mathrm{ml} / \mathrm{ha}+ \\
\mathrm{GM}\end{array}$} & $\begin{array}{c}10.22 \pm \\
3.51\end{array}$ & $\begin{array}{c}18.22 \pm \\
1.00\end{array}$ & $\begin{array}{c}27.56 \pm \\
2.00\end{array}$ & $\begin{array}{c}40.00 \pm \\
2.52\end{array}$ & $\begin{array}{c}67.56 \pm \\
2.89\end{array}$ & $\begin{array}{c}85.78 \pm \\
1.53\end{array}$ & $\begin{array}{c}96.89 \pm \\
3.51\end{array}$ & $\begin{array}{c}100 \pm \\
0.00\end{array}$ & & & \\
\hline & $(2.67)$ & $(7.11)$ & $(10.22)$ & $(10.67)$ & $(11.56)$ & & & & & & \\
\hline Control & $\begin{array}{c}1.78 \pm \\
0.58\end{array}$ & $\begin{array}{c}3.11 \pm \\
0.58\end{array}$ & $\begin{array}{c}3.11 \pm \\
0.00\end{array}$ & $\begin{array}{c}3.56 \pm \\
0.00\end{array}$ & & & & & & & \\
\hline
\end{tabular}

\pm Standard deviation (SD)

( ) \% cannibalised nymphs observed after treatment

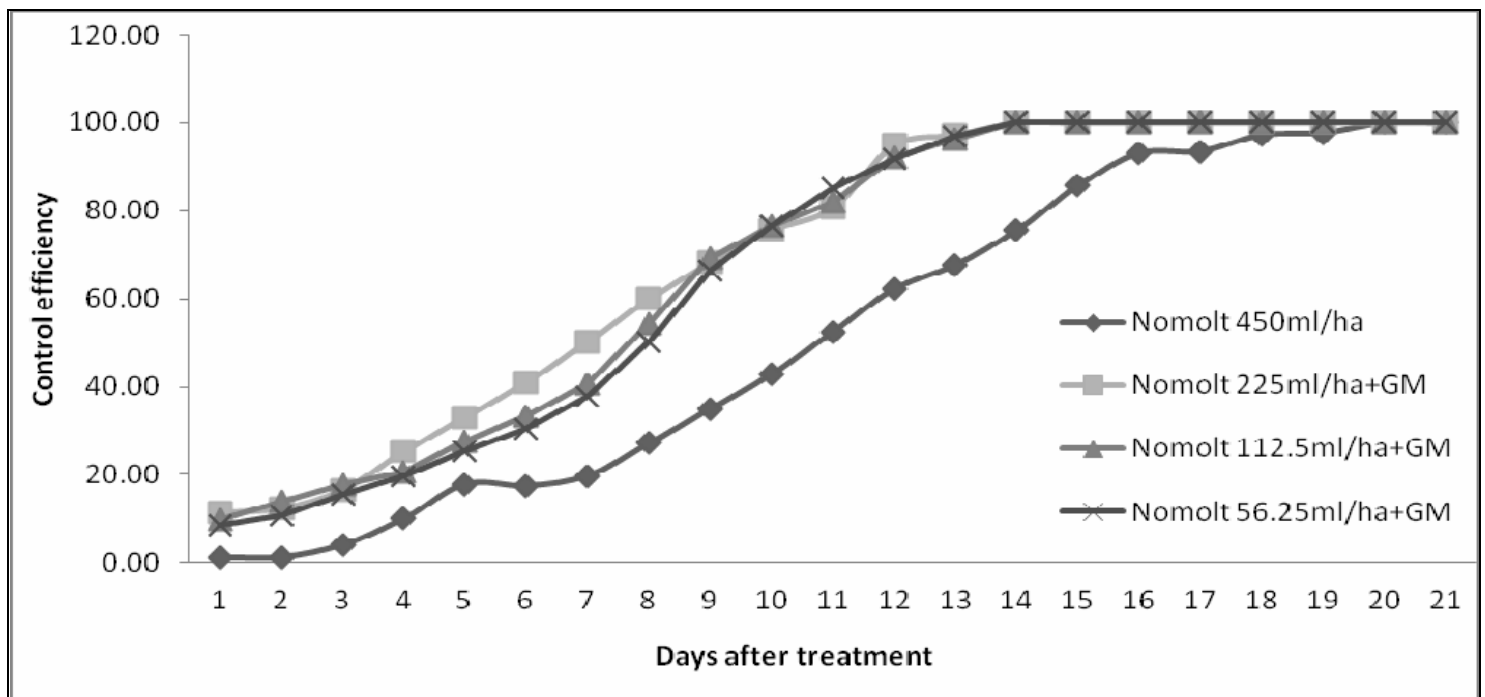

Fig 3. Mean control efficiency of desert locust nymphs treated with different doses of the IGR Nomolt plus GM50g/ha (year/2008) 
Most of the cadavers showed typically GM symptoms which comprise dry cadaver and red color.

In season 2 the fractional doses of Nomolt (225, 112.5 and $56.25 \mathrm{ml} / \mathrm{ha}$ ) combined with GM, $100 \%$ mortality was achieved in 18, 17, and 15 days after treatment respectively. Out of this $100 \%$ mortality, $91.1,86.7$ and $81.3 \%$ was due to treatment effected by the Nomolt combined with GM, and 8.9, 13.3 and $18.7 \%$ due to cannibalism (Table 4). The control efficiency of the control agents are illustrated in Figure 4.

Table 4. \% Mortality of desert locust nymphs caused by various doses of the IGR Nomolt combined with Metarhizium GM (year/2009)

\begin{tabular}{|c|c|c|c|c|c|c|c|c|c|c|c|}
\hline \multirow{2}{*}{ Treatment } & \multicolumn{11}{|c|}{$\%$ Mortality at days after treatment } \\
\hline & 1 & 3 & 5 & 7 & 9 & 11 & 13 & 15 & 17 & 19 & 21 \\
\hline \multirow[t]{2}{*}{$\begin{array}{l}\text { Nomolt } \\
450 \mathrm{ml} / \mathrm{ha}\end{array}$} & $\begin{array}{l}7.56 \pm \\
3.06\end{array}$ & $\begin{array}{c}23.11 \pm \\
3.51\end{array}$ & $\begin{array}{c}46.67 \\
\pm \\
4.93\end{array}$ & $\begin{array}{c}65.33 \\
\pm \\
2.65\end{array}$ & $\begin{array}{c}73.78 \pm \\
4.16\end{array}$ & $\begin{array}{c}81.78 \\
\pm \\
1.53\end{array}$ & $\begin{array}{c}91.11 \\
\pm \\
2.31\end{array}$ & $\begin{array}{c}97.78 \\
\pm \\
1.53\end{array}$ & $\begin{array}{l}100 \pm \\
0.00\end{array}$ & & \\
\hline & (3.11) & $(7.56)$ & (9.33) & $\begin{array}{c}(12.89 \\
)\end{array}$ & (12.89) & $\begin{array}{c}(13.33 \\
)\end{array}$ & & & & & \\
\hline \multirow[t]{2}{*}{$\begin{array}{l}\text { Nomolt } \\
225 \mathrm{ml} / \mathrm{ha}+ \\
\text { GM }\end{array}$} & $\begin{array}{c}0.44 \pm \\
0.58\end{array}$ & $\begin{array}{c}8.44 \pm \\
0.58\end{array}$ & $\begin{array}{c}16.89 \\
\pm \\
0.58\end{array}$ & $\begin{array}{c}33.78 \\
\pm \\
1.15\end{array}$ & $\begin{array}{c}54.22 \pm \\
0.58\end{array}$ & $\begin{array}{c}63.56 \\
\pm \\
4.00\end{array}$ & $\begin{array}{c}78.22 \\
\pm \\
1.15\end{array}$ & $\begin{array}{c}94.22 \\
\pm \\
2.00\end{array}$ & $\begin{array}{c}99.11 \\
\pm \\
1.15\end{array}$ & $\begin{array}{l}100 \pm \\
0.00\end{array}$ & \\
\hline & & $(2.67)$ & $(5.78)$ & $(6.67)$ & $(8.89)$ & & & & & & \\
\hline \multirow[t]{2}{*}{$\begin{array}{l}\text { Nomolt } \\
112.5 \mathrm{ml} / \mathrm{ha}+ \\
\mathrm{GM}\end{array}$} & & $\begin{array}{c}11.11 \pm \\
1.00\end{array}$ & $\begin{array}{c}18.22 \\
\pm \\
2.52\end{array}$ & $\begin{array}{c}41.78 \\
\pm \\
2.65\end{array}$ & $\begin{array}{c}65.33 \pm \\
3.00\end{array}$ & $\begin{array}{c}79.11 \\
\pm \\
3.21\end{array}$ & $\begin{array}{c}90.67 \\
\pm \\
3.06\end{array}$ & $\begin{array}{c}97.33 \\
\pm \\
2.31\end{array}$ & $\begin{array}{l}100 \pm \\
2.31\end{array}$ & & \\
\hline & & $(3.56)$ & $(6.22)$ & $(9.78)$ & (13.33) & & & & & & \\
\hline \multirow[t]{2}{*}{$\begin{array}{l}\text { Nomolt } \\
56.25 \mathrm{ml} / \mathrm{ha}+ \\
\mathrm{GM}\end{array}$} & $\begin{array}{l}0.89 \pm \\
1.15\end{array}$ & $\begin{array}{c}12.00 \pm \\
1.15\end{array}$ & $\begin{array}{c}19.56 \\
\pm \\
2.31\end{array}$ & $\begin{array}{c}41.33 \\
\pm \\
1.15\end{array}$ & $\begin{array}{c}71.11 \pm \\
3.51\end{array}$ & $\begin{array}{c}85.33 \\
\pm \\
2.08\end{array}$ & $\begin{array}{c}93.33 \\
\pm \\
1.53\end{array}$ & $\begin{array}{l}100 \pm \\
4.04\end{array}$ & & & \\
\hline & $(0.44)$ & $(6.22)$ & (9.33) & $\begin{array}{c}(13.33 \\
)\end{array}$ & (17.33) & $\begin{array}{c}(18.22 \\
)\end{array}$ & $\begin{array}{c}(18.67 \\
)\end{array}$ & & & & \\
\hline Control & $\begin{array}{c}1.33 \pm \\
1.00\end{array}$ & $\begin{array}{c}3.11 \pm \\
0.58\end{array}$ & $\begin{array}{c}3.56 \pm \\
0.00\end{array}$ & & & & & & & & \\
\hline
\end{tabular}

\pm Standard deviation (SD)

( ) \% cannibalised nymphs observed after treatment

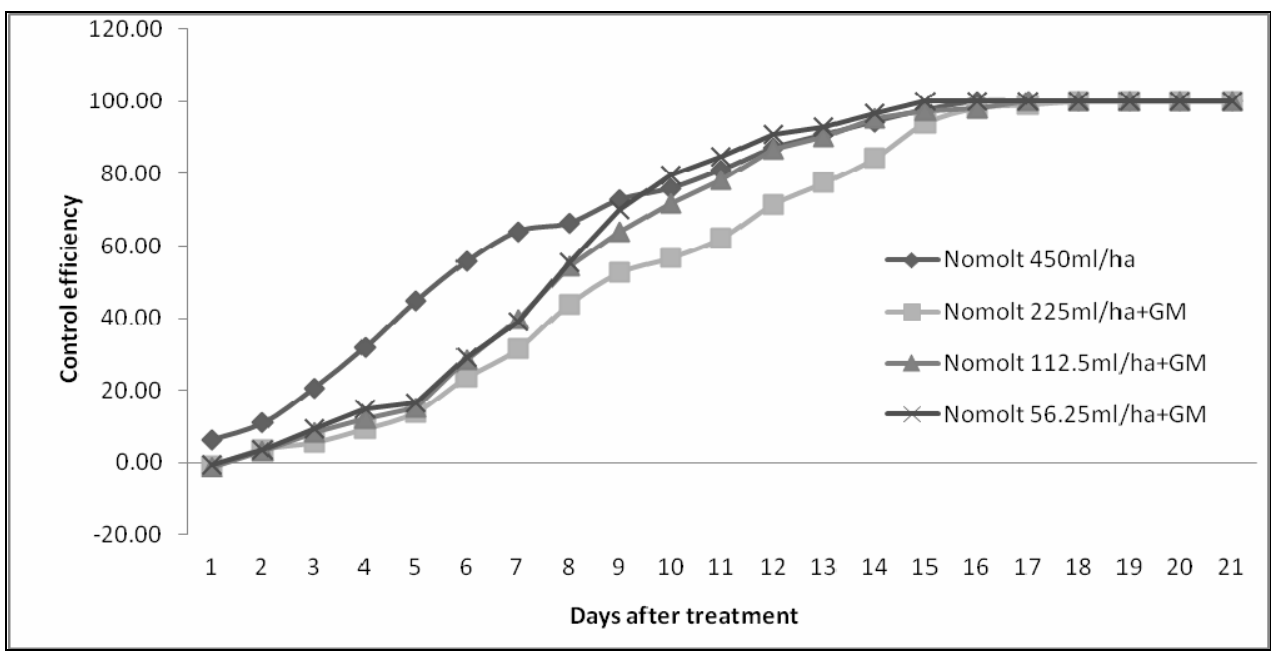

Fig 4. Mean control efficiency of desert locust nymphs treated with different doses of the IGR Nomolt plus Metarhizium GM 50g/ha (year/2009) 
The statistical tests confirmed the effectiveness of the fractional doses of the IGR Nomolt combined with $\mathrm{GM}$, and there was no significant difference with the recommended dose of the Nomolt.

Barrier treatment: Tests in bomas, utilizing Nomolt $450 \mathrm{ml} / \mathrm{ha}$ (recommended dose) as barrier treatment revealed that the total mortality after 21 day, in nymphs exposed to the Nomolt after the $1^{\text {st }}, 6^{\text {th }}, 12^{\text {th }}$ and $24^{\text {th }}$ days after application was $72.5,88.5,89$ and $53 \%$ respectively. Of this $67,54,62.5$ and $52.5 \%$ was due to treatment effected by the Nomolt and percentages $5.5,34.5,26.5$ and 0.5 due to cannibalism (Table 5).

Table 5. \% Mortality of the desert locust nymphs treated with the IGR Nomolt $450 \mathrm{ml} / \mathrm{ha}$ by barrier treatment (year/2008)

\begin{tabular}{|c|c|c|c|c|c|c|c|c|c|c|c|}
\hline \multirow{2}{*}{ Treatment } & \multicolumn{11}{|c|}{$\%$ Mortality at days after treatment } \\
\hline & 1 & 3 & 5 & 7 & 9 & 11 & 13 & 15 & 17 & 19 & 21 \\
\hline \multirow[t]{2}{*}{$\begin{array}{l}1 \text { day after } \\
\text { treatment }\end{array}$} & $\begin{array}{c}2.50 \pm \\
3.54\end{array}$ & $\begin{array}{c}16.00 \pm \\
4.24\end{array}$ & $\begin{array}{c}35.50 \\
\pm \\
6.36\end{array}$ & $\begin{array}{c}50.50 \pm \\
2.83\end{array}$ & $\begin{array}{c}58.50 \\
\pm \\
1.41\end{array}$ & $\begin{array}{c}61.50 \\
\pm \\
1.41\end{array}$ & $\begin{array}{c}62.50 \pm \\
0.71\end{array}$ & $\begin{array}{c}66.00 \\
\pm \\
0.71\end{array}$ & $\begin{array}{c}72.00 \\
\pm \\
1.41\end{array}$ & & \\
\hline & $(2.50)$ & $(5.50)$ & & & & & & & & & \\
\hline \multirow[t]{2}{*}{$\begin{array}{l}6 \text { day after } \\
\text { treatment }\end{array}$} & $\begin{array}{c}2.00 \pm \\
1.41\end{array}$ & $\begin{array}{c}10.50 \pm \\
0.00\end{array}$ & $\begin{array}{c}54.00 \\
\pm \\
17.68\end{array}$ & $\begin{array}{c}64.00 \pm \\
1.41\end{array}$ & $\begin{array}{c}69.00 \\
\pm \\
0.00\end{array}$ & $\begin{array}{c}77.50 \\
\pm \\
1.41\end{array}$ & $\begin{array}{c}80.50 \pm \\
0.71\end{array}$ & $\begin{array}{c}85.00 \\
\pm \\
2.83\end{array}$ & $\begin{array}{c}88.50 \\
\pm \\
0.00\end{array}$ & & \\
\hline & $(2.00)$ & $(10.50)$ & $\begin{array}{c}(34.5 \\
0)\end{array}$ & & & & & & & & \\
\hline \multirow[t]{2}{*}{$\begin{array}{l}12 \text { day after } \\
\text { treatment }\end{array}$} & $\begin{array}{c}21.50 \pm \\
3.54\end{array}$ & $\begin{array}{c}66.00 \pm \\
9.90\end{array}$ & $\begin{array}{c}76.00 \\
\pm \\
0.00\end{array}$ & $\begin{array}{c}81.50 \pm \\
3.54\end{array}$ & $\begin{array}{c}88.00 \\
\pm \\
3.54\end{array}$ & $\begin{array}{c}89.00 \\
\pm \\
0.00\end{array}$ & & & & & \\
\hline & $(21.50)$ & $(26.50)$ & & & & & & & & & \\
\hline \multirow[t]{2}{*}{$\begin{array}{l}24 \text { day after } \\
\text { treatment }\end{array}$} & & $\begin{array}{c}12.00 \pm \\
12.02\end{array}$ & $\begin{array}{c}28.00 \\
\pm \\
3.54\end{array}$ & $\begin{array}{c}37.50 \pm \\
0.71\end{array}$ & $\begin{array}{c}47.00 \\
\pm \\
0.71\end{array}$ & $\begin{array}{c}52.00 \\
\pm \\
0.00\end{array}$ & $\begin{array}{c}52.50 \pm \\
0.71\end{array}$ & $\begin{array}{c}53.00 \\
\pm \\
0.00\end{array}$ & & & \\
\hline & $(0.50)$ & & & & & & & & & & \\
\hline Control & & $\begin{array}{c}2.50 \pm \\
0.71\end{array}$ & $\begin{array}{c}6.50 \pm \\
2.12\end{array}$ & $\begin{array}{c}7.00 \pm \\
0.00\end{array}$ & & & & & & & \\
\hline
\end{tabular}

\pm Standard deviation (SD)

( ) \% cannibalised nymphs observed after treatment

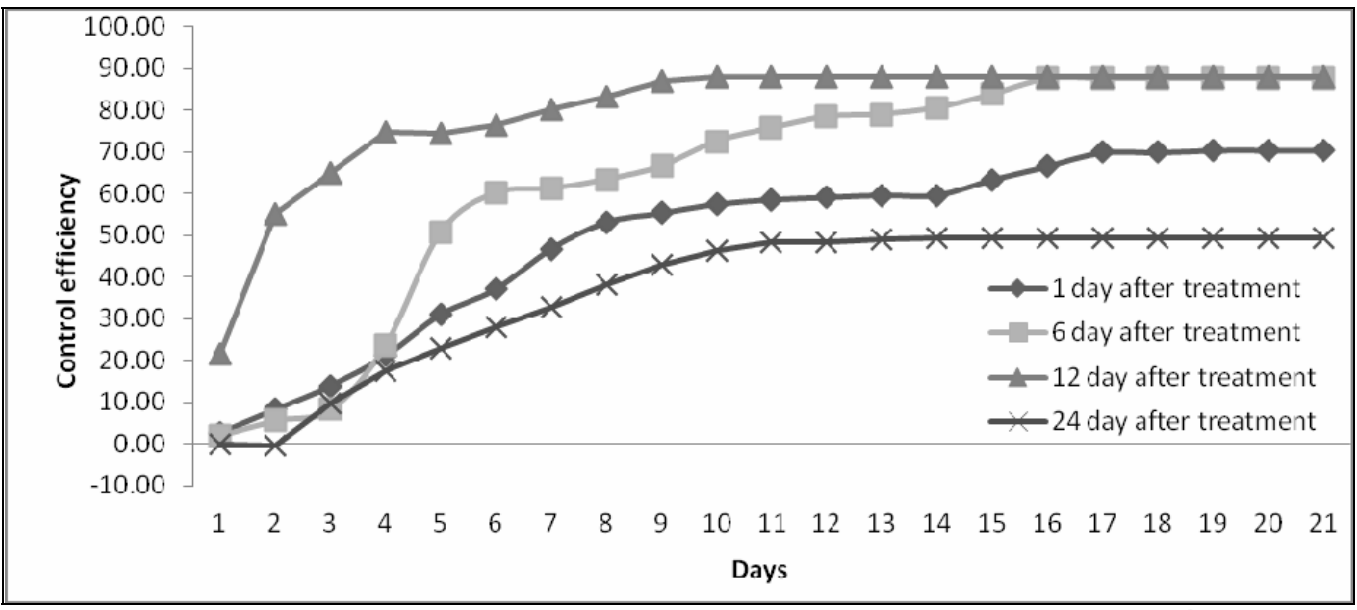

Fig 5. Mean control efficiency of desert locust nymphs treated with the IGR Nomolt $450 \mathrm{ml} / \mathrm{ha}$ by barrier treatment (year/2008) 
The control efficiency of the control agent under investigation is revealed in Fig. 5.

The barrier treatment with the Nomolt $450 \mathrm{ml} / \mathrm{ha}$ during different days of exposure after treatment were effective, and there was no significant difference among the different days after treatment. The 1and 24 day after treatment were not significantly different from the control.

In season 2 tests in bomas, utilizing Nomolt $450 \mathrm{ml} / \mathrm{ha}$ (recommended dose) as barrier treatment showed that the total mortality after 21day, in nymphs exposed to the Nomolt after the $1^{\text {st }}, 6^{\text {th }}, 12^{\text {th }}$, and $24^{\text {th }}$ day after application, was 78.5, 68.5, 74 and $61.5 \%$ respectively. Of this $57,41,59.5$ and $48.5 \%$ was due to treatment effected by the Nomolt, and percentages 21.5, 28.5, 10 and 13 due to cannibalism (Table 6).

The control efficiency of the control agent under investigation is shown in Fig. 6.

Table 6. \% Mortality of desert locust nymphs treated with the IGR Nomolt $450 \mathrm{ml} /$ ha by barrier treatment (year/2009)

\begin{tabular}{|c|c|c|c|c|c|c|c|c|c|c|c|}
\hline \multirow{2}{*}{ Treatment } & \multicolumn{11}{|c|}{$\%$ Mortality at days after treatment } \\
\hline & 1 & 3 & 5 & 7 & 9 & 11 & 13 & 15 & 17 & 19 & 21 \\
\hline \multirow[t]{2}{*}{$\begin{array}{l}1 \text { day after } \\
\text { treatment }\end{array}$} & $\begin{array}{c}1.00 \pm \\
1.41\end{array}$ & $\begin{array}{c}3.00 \pm \\
2.12\end{array}$ & $\begin{array}{c}6.00 \pm \\
0.71\end{array}$ & $\begin{array}{c}24.00 \\
\pm \\
2.12\end{array}$ & $\begin{array}{c}46.00 \pm \\
2.12\end{array}$ & $\begin{array}{c}51.00 \\
\pm \\
0.00\end{array}$ & $\begin{array}{c}68.00 \pm \\
1.41\end{array}$ & $\begin{array}{c}76.00 \\
\pm \\
0.00\end{array}$ & $\begin{array}{c}76.50 \\
\pm \\
0.71\end{array}$ & $\begin{array}{c}77.00 \pm \\
0.00\end{array}$ & $\begin{array}{c}78.50 \\
\pm \\
0.71\end{array}$ \\
\hline & $(0.00)$ & $(0.50)$ & $(2.00)$ & $(7.00)$ & $(12.50)$ & $\begin{array}{c}(13.50 \\
)\end{array}$ & $(20.50)$ & $\begin{array}{c}(21.50 \\
)\end{array}$ & & & \\
\hline \multirow[t]{2}{*}{$\begin{array}{l}6 \text { day after } \\
\text { treatment }\end{array}$} & $\begin{array}{c}3.50 \pm \\
3.54\end{array}$ & $\begin{array}{c}10.50 \\
\pm \\
4.24\end{array}$ & $\begin{array}{c}20.00 \\
\pm \\
12.02\end{array}$ & $\begin{array}{c}47.50 \\
\pm \\
2.12\end{array}$ & $\begin{array}{c}61.00 \pm \\
2.83\end{array}$ & $\begin{array}{c}64.50 \\
\pm \\
2.12\end{array}$ & $\begin{array}{c}65.00 \pm \\
0.00\end{array}$ & $\begin{array}{c}65.00 \\
\pm \\
0.00\end{array}$ & $\begin{array}{c}66.50 \\
\pm \\
0.00\end{array}$ & $\begin{array}{c}68.50 \pm \\
0.00\end{array}$ & \\
\hline & (1.50) & $(6.00)$ & $(7.50)$ & $\begin{array}{c}(21.00 \\
)\end{array}$ & $(28.00)$ & & & & & & \\
\hline \multirow[t]{2}{*}{$\begin{array}{l}12 \text { day after } \\
\text { treatment }\end{array}$} & $\begin{array}{c}5.50 \pm \\
0.71\end{array}$ & $\begin{array}{c}32.50 \\
\pm \\
3.54\end{array}$ & $\begin{array}{c}59.50 \\
\pm \\
2.12\end{array}$ & $\begin{array}{c}68.50 \\
\pm \\
3.54\end{array}$ & $\begin{array}{c}68.50 \pm \\
0.00\end{array}$ & $\begin{array}{c}71.00 \\
\pm \\
0.00\end{array}$ & $\begin{array}{c}73.50 \pm \\
0.00\end{array}$ & $\begin{array}{c}74.00 \\
\pm \\
0.00\end{array}$ & & & \\
\hline & $(0.00)$ & (1.50) & (3.50) & $(9.00)$ & $(9.00)$ & $(9.00)$ & $(10.00)$ & & & & \\
\hline \multirow[t]{2}{*}{$\begin{array}{l}24 \text { day after } \\
\text { treatment }\end{array}$} & $\begin{array}{c}6.00 \pm \\
1.41\end{array}$ & $\begin{array}{c}22.50 \\
\pm \\
2.83\end{array}$ & $\begin{array}{c}47.00 \\
\pm \\
2.83\end{array}$ & $\begin{array}{c}51.00 \\
\pm \\
0.00\end{array}$ & $\begin{array}{c}51.00 \pm \\
0.00\end{array}$ & $\begin{array}{c}53.50 \\
\pm \\
0.71\end{array}$ & $\begin{array}{c}57.50 \pm \\
0.71\end{array}$ & $\begin{array}{c}58.00 \\
\pm \\
0.00\end{array}$ & $\begin{array}{c}61.50 \\
\pm \\
0.00\end{array}$ & & \\
\hline & $(1.50)$ & $(4.00)$ & $\begin{array}{c}(11.50 \\
)\end{array}$ & $\begin{array}{c}(11.50 \\
)\end{array}$ & $(11.50)$ & $\begin{array}{c}(12.50 \\
)\end{array}$ & $(13.00)$ & & & & \\
\hline Control & $\begin{array}{c}0.50 \pm \\
0.71\end{array}$ & $\begin{array}{c}2.50 \pm \\
0.71\end{array}$ & $\begin{array}{c}4.50 \pm \\
1.41\end{array}$ & $\begin{array}{c}5.00 \pm \\
0.00\end{array}$ & $\begin{array}{c}5.00 \pm \\
0.00\end{array}$ & $\begin{array}{c}5.50 \pm \\
0.71\end{array}$ & $\begin{array}{c}6.00 \pm \\
0.00\end{array}$ & & & & \\
\hline
\end{tabular}

\pm Standard deviation (SD)

( ) \% cannibalized nymphs observed after treatment

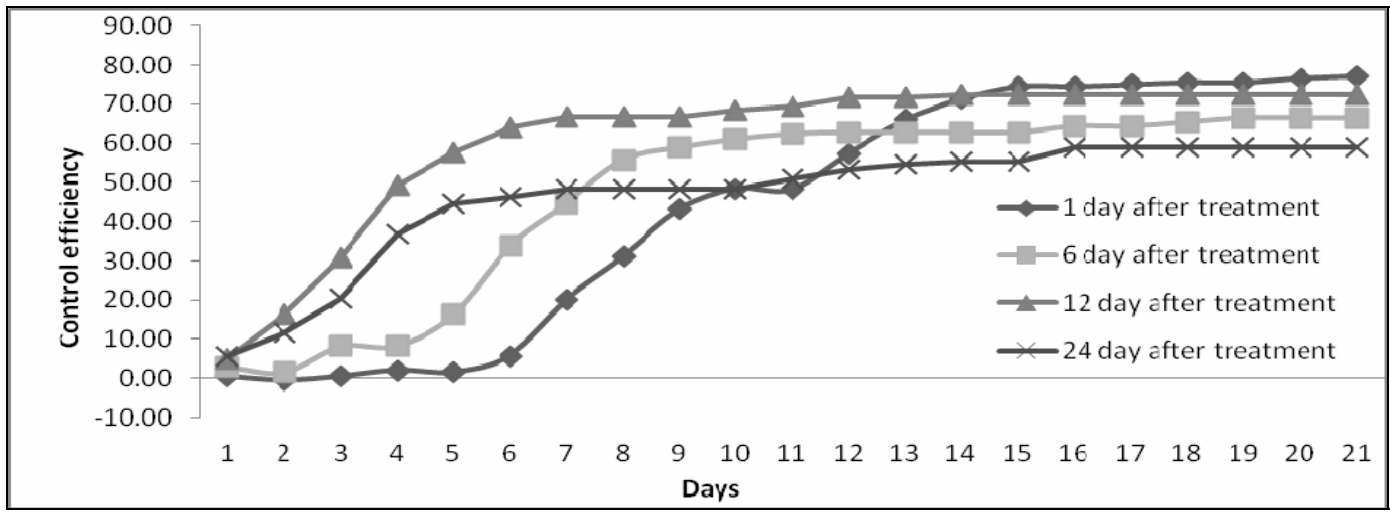

Fig 6. Mean control efficiency of desert locust nymphs treated with the IGR Nomolt 450ml/ha by barrier treatment (year/2009) 
There was no significant difference among the different days after treatment; however they were significantly different from control.

\section{DISCUSSION}

The use of the fractional doses $(225 \mathrm{ml} / \mathrm{ha}$, $112.5 \mathrm{ml} / \mathrm{ha}$ and $56.25 \mathrm{ml} / \mathrm{ha}$ ) of the IGR Nomolt in combination with pheromone PAN $10 \mathrm{ml} / \mathrm{ha}$, proved its efficacy on the DL nymphs, and in all cases superseded the effect of the recommended dose alone. This can reflect positively on reducing cost and environment hazards, particularly the quantity of the pheromone used in desert locust manipulation is very low, the recommended dose $10 \mathrm{ml} / \mathrm{ha}$ i.e. less than 40 cents/ha (Rono et al., 2008). Although semiochemicals have been successfully used in the field for the management of some pests, they are not generally considered to be sufficiently reliable in their action when used alone. They are effective when used as part of integrated control strategies (Smart et al., 1994). The results indicated that a combination of the IGR Nomolt and GM enhanced the speed of action, and high mortality could be obtained with lower doses of Nomolt. The differences in the results of the trials during the two seasons could be attributed to the variation in weather factors such as temperature and relative humidity, particularly when the IGR Nomolt plus GM were tested. Field trials conducted by Hunter et al., (1999) and (2001), provided clear evidence of the efficacy of mycopesticides based on M. anisopliae var. acridum, depending on climatic conditions, the extreme day/night temperature fluctuations may impede fungal pathogenesis and increase the chances of survival in infected locusts (Peveling, 2007).

The combination of the fractional doses of the IGR Nomolt with GM revealed more effectiveness, compared with the rest of the preparations. These findings agree with that report by Hassan and Charnley (1989), Joshi et al. (1992) who argued that IGRs can weaken the cuticle and reduce resistance to fungal penetration. The IGR Nomolt proved to be more effective against DL nymphs as barrier treatment due to its long persistence. This can be reflected positively on reducing cost and environmental hazards. These results have been achieved within bomas with limited space and restriction of the movement of the test nymphs. Tests under real infestation and field conditions are needed to verify these results; however, Zehrer (1997) showed that insecticides represent the greatest cost factor in the control of locusts and grasshoppers in Madagascar. By using insect growth regulators in barrier treatment, control costs can be reduced by half. For example US $\$ 2.30 /$ ha could be saved if 4000 ha were treated by this method. Bouaichi et al., (1994) also noted that barrier spraying allows large areas to be treated quickly and cheaply by spraying strips up to $2 \mathrm{~km}$ apart. Four parameters - spray swath, swath separation and the concentration and persistence of pesticide - all affect the outcome of barrier spraying. Insect growth regulators demonstrate both persistence and some selectivity. Diflubenzuron is one example of chitin synthesis inhibitors (Reynolds, 1987) that has up to six weeks, persistence in sahelian grassland (Sissoko, 1991). The Nomolt has greater persistence, and is suitable for barrier treatment and should be applied against early hopper instars; however, it is not suitable for crop protection due to its slow action.

Tests have demonstrated the reduction in food consumed even at fractional doses of the IGR Nomolt when used alone or combined with pheromone PAN and GM. However, the combination of the IGR Nomolt with GM revealed clear effect on feeding rate of DL nymphs comparing with other formulations. These finding agreed with Grosscurt (1978), Clark and Jewess (1990) who indicated that the Nomolt showed reduction of feeding and mobility and highly significant differences between treated and control cages were found.

\section{REFERENCES}

Bouaichi, A., Coppen, G. D. A. and Jepson, P. C.(1994) Barrier spray treatment with diflubenzuron (ULV) against gregarious hopper bands of the Moroccan locust, Dociostaaurus maroccanus (Thunberg) (Orthoptera: Acrididae) in Morocco, Crop Protection 1994 vol. 13no. 1.

Ceccato, P., Cressman, K., Giannini, A. and Trzaska, S. (2007) The desert locust upsurge in West Africa (20032005): Information on the desert locust early warning system and the prospects for seasonal climate forecasting. Intl J Pest Management 53(1): 7-13

Clarke, B.S. and Jewess, P.J. (1990) The inhibition of chitin synthesis in Spodoptora littoralis larvae by flufenoxyron, teflubenzuron and diflubenzuron. Pesticide Science 28: 377 - 388

Grosscurt, A. C. (1978) Diflubenzuron: Some aspects of its ovicidal and larvicidal mode of action and an evaluation of its practical possibilities. Pestic. Sci. 9, 373-386.

Hassan, A. E. M. and Charnley, A. K. (1989) Ultrastrucural study of the penetration by Metarhizium anisopliae through Dimilin affected cuticle of Manduca sexta. J. Invert. Pathol. 54, 117-124. 
Henderson, C.F. and Tilton, E.W. (1955) Tests with acaricides against the brown wheal mite. J. economic entom. 48, 159-161.

Hunter, D.M., Milner, R.J., Scanlan, J.C. and Spurgin, P.A. (1999) Aerial treatment of the migratory locust, Locusta migratoria (L.) (Orthoptera: Acrididae) with Metarhizium anisopliae (Deuteromycotina: Hyphomycetes) in Australia. Crop Protection 18: 699704.

Hunter, D.M., Milner, R.J. and Spurgin, P.A. (2001). Aerial treatment of the Australian plague locust, Chortoicetes terminifera (Orthoptera: Acrididae) with Metarhizium anisopliae (Deuteromycotina: Hyphomycetes). Bulletin of Entomological Research 91: 93-99.

Joshi, L., Charnley, A. K., Arnold, G., Brain, P. and Bateman (1992) Synergism between entomopathogenic fungi Metarhizium species and the benzoylurea insecticide, teflubenzuron, against the desert locust Schistocerca gregaria. Proc. Bri. Crop Prot. Conf. 1, 369-374.

Lecoq, M., Mestre, J. and Rachadi, T. (1988) Essai au Tchad d'un inhibiteur de croissance pour la lute contre le croquet pelerin Schistocerca gregaria. D.310 CIRAD-PRIFAS, Montpellier, France, p. 47.

Müller, P. (1988) Okotoxikologische Wirkungen von chloriertenKohlenwasserstoffen, Phosphorsäureestern, Carbamaten und Pyrethroiden im nordöstlichen Sudan. Unpubl. Report to GTZ. Eschborn, Saarbrücken. $70 \mathrm{pp}$

Peveling, R., Weyrich, J. and Müller, P. (1994) Side-effects of botanicals, insect growth regulators and entomopathogenic fungi on epigeal non-target arthropods in locust control In: New Trends in Locust Control (eds. S. Krall, H. Wilps), GTZ, Eschborn, TZVerlagsgesellschaft Rossdorf, p. 148-176

Peveling, R. (2007) Evidence versus perception in locust control, Journal of Orthoptera Research 2005,14(2): 207-212.

Rembold, H. (1994) Controlling locusts with plant chemicals In: New Trends in Locust Control (eds. S. Krall, H. Wilps), GTZ, Eschborn, TZVerlagsgesellschaft Rossdorf, p. 41-49

Reynolds, S.E. (1987) The cuticle, growth and moulting in insects: the essential background to the action of acylurea insecticides. Pesticide Science 20: 131 - 146

Rono, E., Njagi, P.G.N., Bashir, M.O., and Hassanalia A. (2008) Concentration-dependent parsimonious releaser roles of gregarious male pheromone of the desert locust, Schistocerca gregaria. Journal of Insect Physiology 54/162-168.

Sissoko, M. (1991) A novel experimental investigation of the persistence of dimilin against grasshoppers. MPhil. Thesis, University of Southhampton, UK.

Smart, L.E., Blight, M.M., Pickett, J.A. and Pye, B.J. (1994) Development of field strategies incorporating semiochemicals for the control of the pea and bean weevil, Sitona lineatus L. Crop Protection 13: 127 135

Zehrer, W. (1997) Comparison of the costs of barrier and blanket treatments using insect growth regulators in Madagascar In: New Strategies in Locust Control (eds. S. Krall, R. Peveling, D. Ba Diallo), Birkhäuser Verlag, Basel,

p.

425-432 